\title{
SLAM-based Indoor Navigation in University Buildings
}

\author{
Ekaterina Sukhareva ${ }^{1}$, Tatiana Tomchinskaya ${ }^{l}$ and Ilya Serov ${ }^{1}$ \\ ${ }^{1}$ NNSTU n.a. R.E. Alekseev, Minin Street, 24, Nizhny Novgorod, 603950, Russia
}

\begin{abstract}
The article discusses the use of SLAM (simultaneous localization and mapping) technology, with the help of which it is possible to build Indoor navigation systems using augmented reality technology, including on mobile platforms. The article also provides an overview of the positive and negative aspects of the SLAM technology its principle of operation for positioning a navigator using augmented reality in a university building within the framework of a student project are reviewed. The already implemented projects on similar topics, but using other technologies are considered their features are described. An example of the implementation of an indoor positioning system in a university using SLAM is given.
\end{abstract}

\section{Keywords}

Augmented reality, indoor navigation, ARCore, SLAM, Unity

\section{Introduction}

Indoor navigation is the navigation and detection of objects (devices or people) inside a building using technologies different from the usual GPS, since GPS is not able to determine the location of the device with the required accuracy within the same building.

Nowadays there are many huge and complexly structured buildings, where it is very easy to get lost or not have time to find the right place in time. Imagine you are late for your plane and cannot find the desired terminal in a large airport or that five minutes are left before an important interview and you are lost in the office building of the company and cannot find the office you need for the interview in time, or that you are a freshman student and you are late for an important exam with a strict teacher who does not tolerate tardiness, but you do not know where the audience is, where the exam is taking place. In such and many other life situations Indoor navigation systems come to the rescue. Often these systems are simply a map of the floors of a building on which a route can be plotted and followed. However, what to do for those who have problems with the perception of cards or a person simply may not have time to figure out where to follow, since his head is already crammed with an important interview or maybe even completely because of the haste and nervous state, the person could turn to the wrong side where the route led? In such situations, indoor navigation systems with using augmented reality can come to the rescue. The main idea of such systems is the interactive display of the route on the user's smartphone camera. That is a person simply launches the application, holds the camera in front of him and the arrows appear on the screen, which he follows. As you move, the arrows are updated and thus the person arrives at the destination on time (Figure 1).

The purpose of the article is to consider some methods of determining the location inside a building, in particular to discuss in details the Simultaneous Localization and Mapping method (Simultanious localization and mapping) as well as to give examples of the implementation of such systems including an application for determining the location using SLAM on floor of the NSTU building [1-6].

GraphiCon 2021: 31st International Conference on Computer Graphics and Vision, September 27-30, 2021, Nizhny Novgorod, Russia EMAIL: suharevakati@gmail.com (E. Sukhareva); tomchinskaya@mail.ru (T. Tomchinskaya); ilyaserov07@gmail.com (I. Serov) ORCID: 0000-0003-4056-6116 (E. Sukhareva); 0000-0002-4693-7478 (T. Tomchinskaya); 0000-0002-6397-9629 (I. Serov)

$\begin{array}{ll}\text { (c) (i) } & \text { (c) } 2021 \text { Copyright for this paper by its authors. } \\ \text { Use permitted under Creative Commons License Attribution } 4.0 \text { International (CC BY 4.0). } \\ \text { CEUR Workshop Proceedings (CEUR-WS.org) }\end{array}$ 

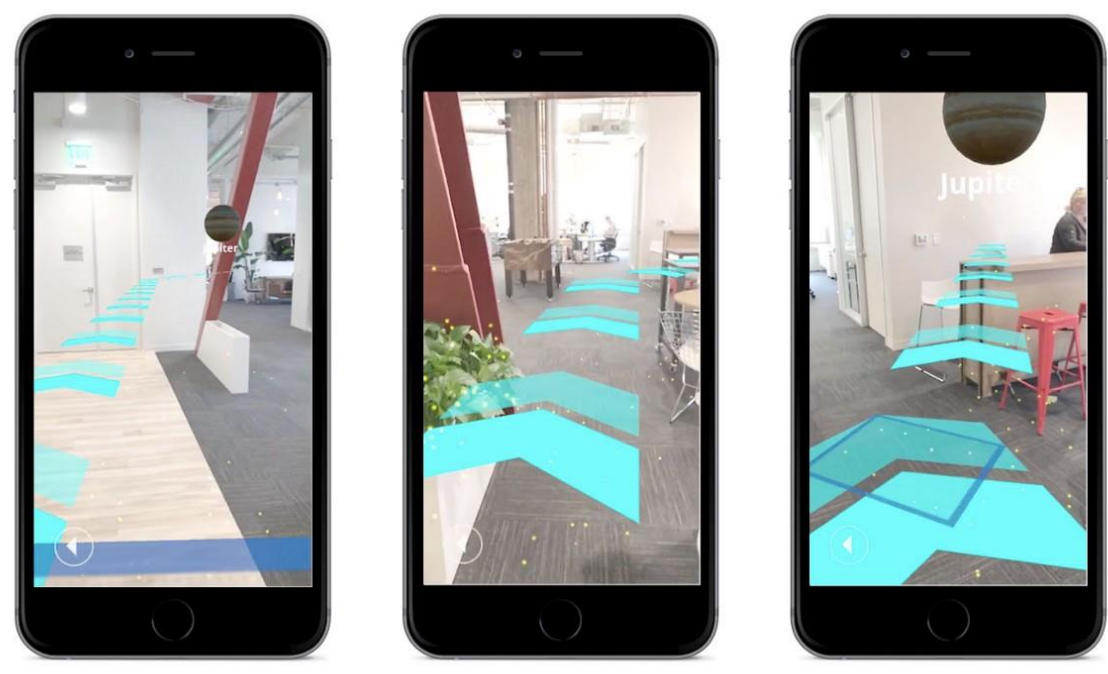

Figure 1: An example of an Indoor navigation system using augmented reality technology

\section{Locating with SLAM}

The most important aspect when building indoor navigation systems using augmented reality is determining the location of the device inside the building. Bluetooth Low Energy iBeacon technology is often used for this. Another popular location technology is RSSI Fingerprinting. This technology uses Wi-Fi signals to build radio maps. However, both of these technologies require material and physical costs for realization. Another interesting technology that allows determining and tracking the location of a device inside a building is SLAM or Simultanious Localization and Mapping. The essence of the SLAM technology is that it is necessary to build a map of the unknown environment and to localize the position of the sensor on this map in real time (the algorithm must have time to work out 30 times per second). The primary sensor for SLAM algorithms is the device camera, as it provides extensive information about the environment with minimal overhead. In addition to the camera, the device uses a gyroscope and accelerometer. SLAM algorithms are available in Google's ARCore development toolkit, similar to Apple's ARCore is ARKit. On the Internet, there are a few examples of creating applications and indoor navigation systems with augmented reality which were created exactly with the help of these tools [7-9].

Consider ARCore, Google's platform for building augmented reality applications without the need for in-depth knowledge of OpenGL and rendering. ARCore uses several key technologies to bring augmented reality to the real world:

1. tracking movements - with the help of this the smartphone can understand its position in the environment;

2. understanding the aspects of the surrounding reality - allows you to determine the location,

size and other details of surrounding surfaces and objects;

3. illumination assessment - allows the device to assess the ambient lighting conditions.

So how does ARCore work? With the help of the device's camera when the user moves his smartphone smart algorithms create their own virtual space with objects based on the real environment. That is why when, in other augmented reality applications, users place virtual objects in the room, go out and come back, their created objects remain in place. ARCore has built its own virtual world in which it remembers where every detail is situated. Google ARCore SDK can be used for free, but with a mandatory mark in the application for using this tool. Most often, ARCore is used in conjunction with the Unity engine.

SLAM algorithms have their own advantages and disadvantages for their using in indoor positioning systems. Their most important advantage is that they do not require any preliminary actions and preparation as in the cases of using Wi-Fi radio cards and BLE (Bluetooth Low Energy) beacons. The disadvantage of this technology is the instability of work in places: 
- $\quad$ with a lot of moving objects;

- poorly lit;

- with the same type of surroundings.

University buildings can be attributed to such places, because basically they are many of the same type of corridors with many moving people [10-11].

\section{Examples of indoor navigation systems with augmented reality}

1. Creating an ARCore powered indoor navigation application in Unity. An example of navigating an office and several adjoining rooms. In this implementation, the SLAM algorithms were used in the implementation from Google ARCore in conjunction with Unity. First, a map of the premises for navigation was compiled here, after which it was transferred to Unity at a 1: 1 scale with the real world, the blue pointer marked with a red rectangle is a location marker that will move in accordance with the change in the location of the device (Figure 2).

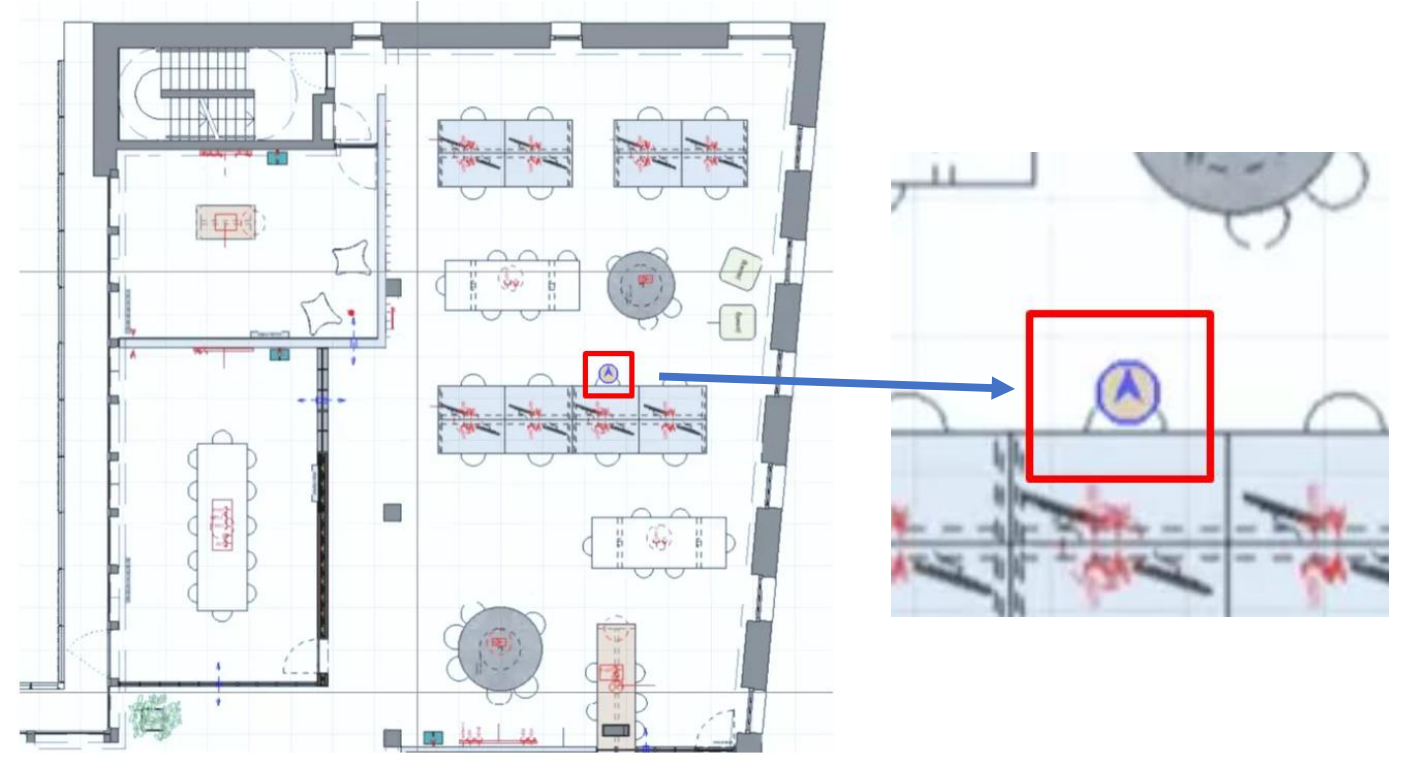

Figure 2: Map for navigation in the example "Creating an ARCore powered indoor navigation application in Unity"

A QR code is used to determine the starting position and synchronization, and the further position is calculated using SLAM. In this example, the use of such a method is explained by the fact that when the application exits, the ARCore functions are "disabled", and in order to "enable" them, it is necessary to perform primary localization. It also points out that to improve navigation performance, more QR codes can be added around the perimeter of the navigation terrain so that the location of the blue pointer can be adjusted from time to time to a more accurate position. The idea of using QR codes is quite popular in the field of Indoor navigation. This is a pretty good way to improve the quality of navigation within a university campus, as it is budgetary and realizable [12].

This example uses NavMesh to find the best route. NavMesh is Unity's built-in navigation surface generator. This is a good free tool for building routes for various navigation tasks in Unity.

The author of the project, in the conclusions about the work done notes that the system is far from perfect, he also thinks about ways to improve the project.

2. Overcoming the problem of uncertain tracking errors in an AR navigation application. Another example of building an indoor navigation system using augmented reality. This project is notable for the fact that an indoor navigation system is being created here in the university building. A 1: $13 \mathrm{D}$ model of the campus building was created in Unity to calculate navigation-related data such as routes or destinations, and to create augmented reality guides (Figure 3 ). 


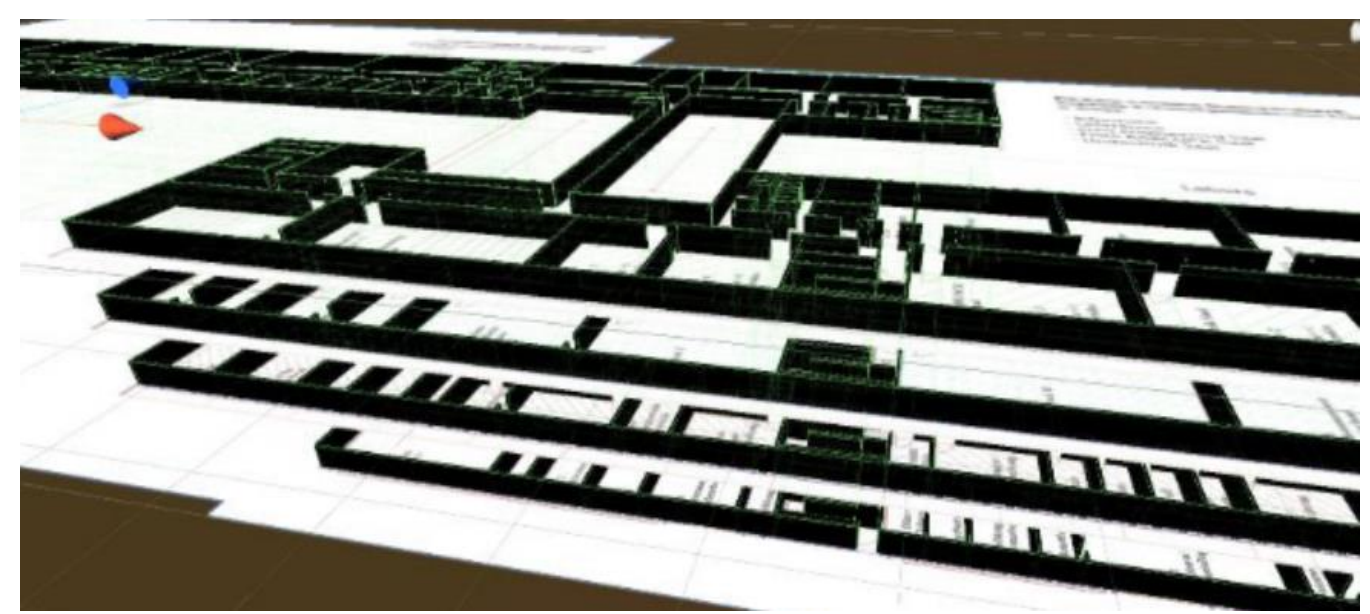

Figure 3: 3D model of the university campus from the second example

ARCore tools were used to work with elements of augmented reality and to create a virtual network of connections. As in the previous example, the NavMesh Navigation Surface Generator was used to create navigation areas. The author notes that there was a need to store audience data, it was necessary to create a database to store all the information. The selected data storage method is to create a JSON file with two data groups: "Room name" and "Description". As a solution to the problem of the instability of calculating the user's location, an approach similar to the one proposed in the previous example with QR codes is used, however, here instead of codes, signs on the auditoriums are used as markers. We use a method for optical recognition based on the Google Cloud Vision API which requires a private key. Of course, it is possible to use free analogs of optical recognition systems here, but it is not known how well they will work, or you can use the same QR codes. Figure 4 shows what the final version of the application from this example looks like [13].

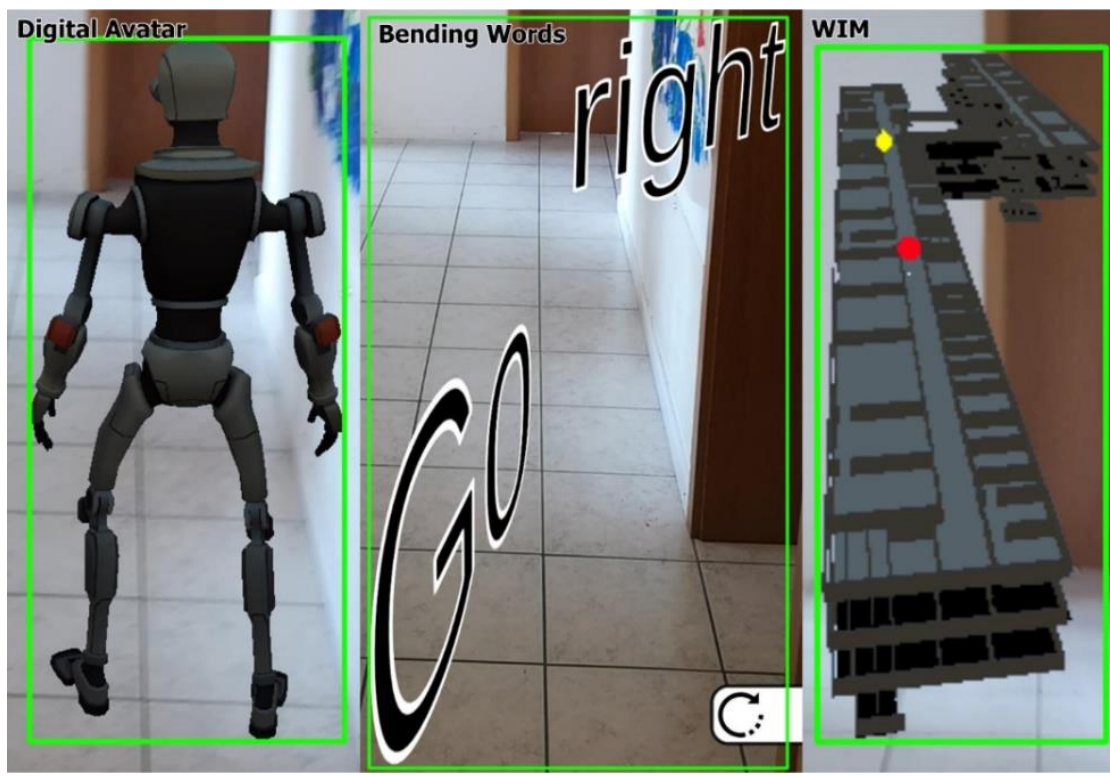

Figure 4: The final version of the AR navigation application

\section{Creation of a prototype application for determining location using SLAM technology on the floor in the building of NSTU}

To create an application for navigating the floor of the NSTU building, SLAM was chosen as a technology for determining the location of a smartphone, since this technology is capable to give a very accurate result under good conditions and also does not require extra costs for implementation. The principle of operation of this system is similar to the first of the examples described above, namely: the 
application was created using Unity + ARCore, to display the movement of the user and his device, a map of the floor of the campus building at a scale of 1: 1 is used, a pointer with an arrow indicating the user (Figure 5-6. Application interface for locating on the campus floor).

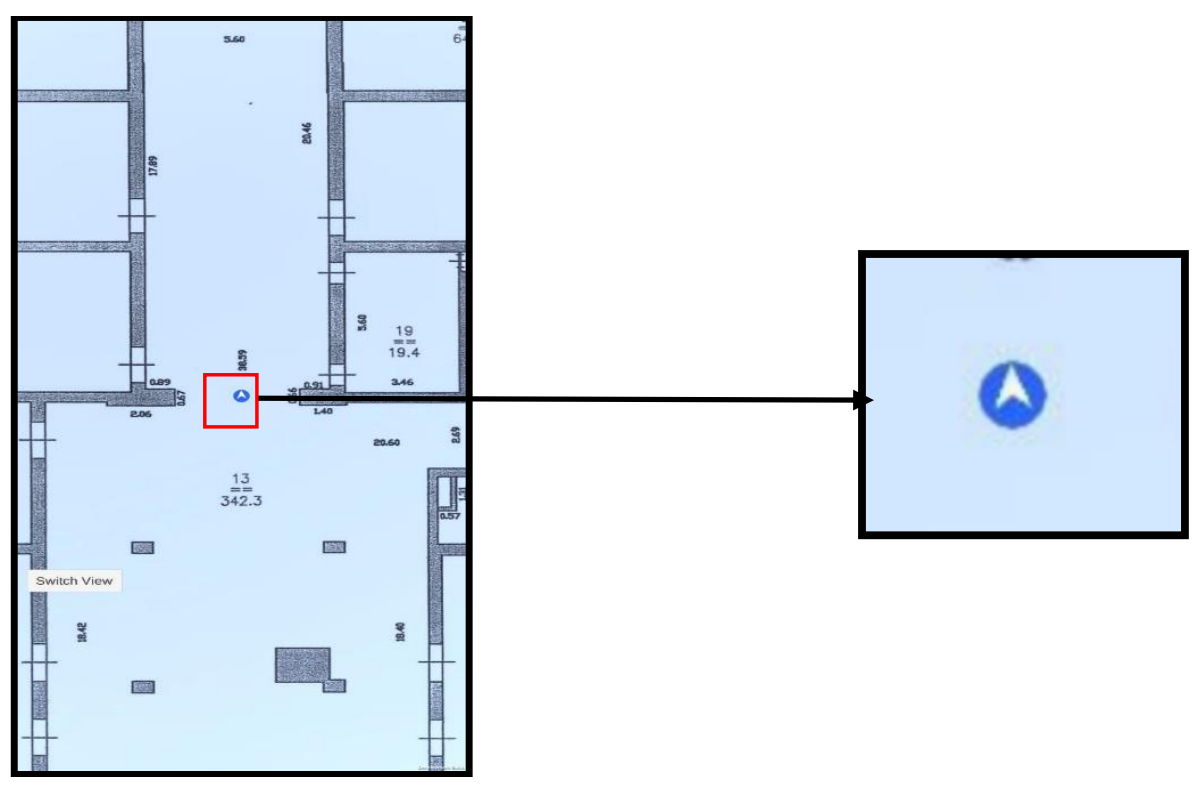

Figure 5: Determination of the location on the floor of the NSTU campus (map view) The location indicator is highlighted in red

More accurate synchronization of the original location was achieved by reading the data about the names of special objects set on the map in Unity from a QR code. To quickly and easily read QR codes, the ZXing library was used.

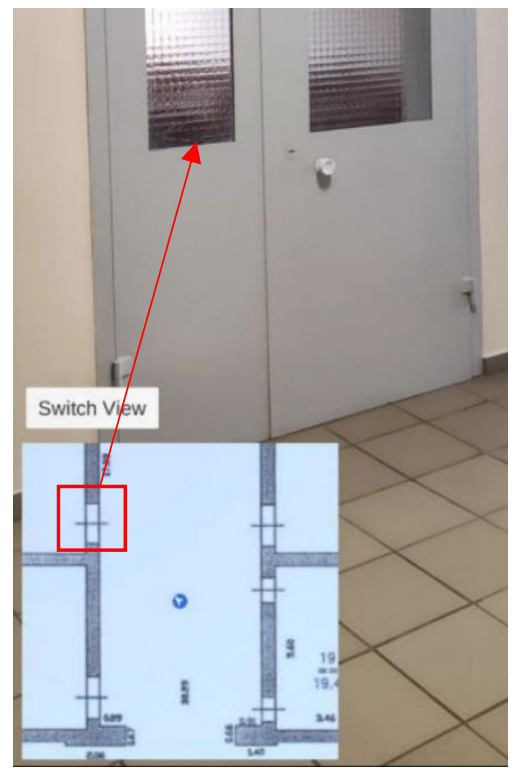

Figure 6a: Application interface for determining the location on the floor of the NSTU campus (camera view) 


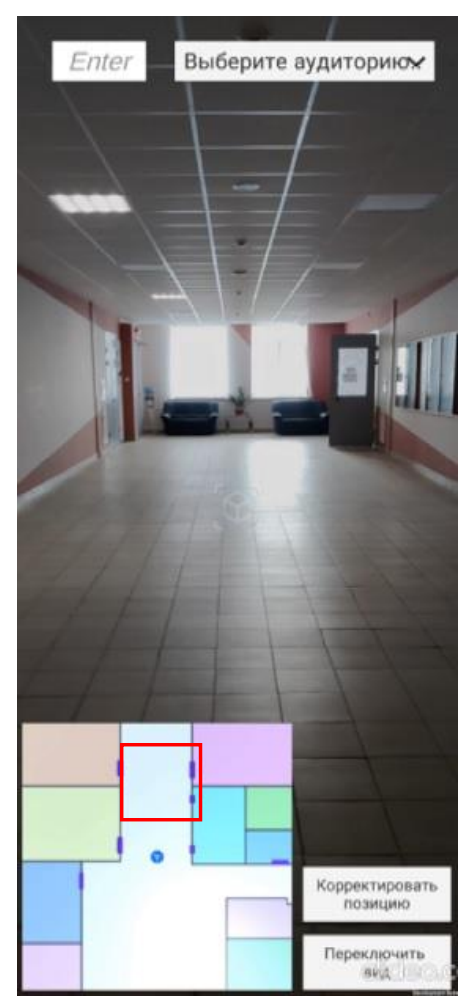

Figure 6b: Application interface for determining the location on the floor of the NSTU campus (camera view)

When testing the application, SLAM technology showed its strengths and weaknesses. In well-lit locations and with the smooth movement of the smartphone, the location is determined very accurately, up to centimeters. In dark places or when the smartphone moves very quickly (blur appears in the camera) errors occur most often: the rotation of the device is not read, that is why further navigation is simply impossible. Such errors can be eliminated by adding more QR codes around the entire perimeter of the map with which to synchronize the location from time to time. In general, SLAM is one of the most suitable and really well working technologies for determining the location of a smartphone inside a university building.

\section{Conclusion}

Based on the considered technologies and examples, we can conclude that building an indoor navigation system using augmented reality in a university with minimal material costs - possibly using SLAM technology. However, it cannot be guaranteed that such a system will work perfectly, or at least well, since the SLAM technology itself is quite young and has many disadvantages. Despite this, there are already various methods to circumvent SLAM imperfections and errors - for example, the use of visual tags or QR codes around the entire perimeter of the building.

\section{References}

[1] A.V. Mongush, P.M. Kikin, Overview of indoor navigation technologies, in: Proceedings of the 14th. International exhibition and scientific congress Interexpo GEO-Siberia, GEO-Siberia'9(1), Novosibirsk, 2017, pp. 119-123.

[2] S.V. Aleshin, Comparative analysis of existing indoor navigation systems for mobile robots for solving the SLAM problem, in: Proceedings of the 6th. Scientific-practical conference "Science of the present and the future", St. Petersburg, 2018, pp. 285-289. 
[3] A.V. Mezhenin, K.I. Gulyaeva, Methods for constructing Indoor-navigation, in: Proceedings of the 46th. scientific and educational conference, Almanac of scientific works of young scientists of ITMO University, St. Petersburg, 2017, pp. 206-209.

[4] M. Harvin, Indoor Positioning System - High-precision Positioning System Inside the Premises. URL:http://blog.jammer.su/2014/02/indoor-positioning-system-pozicionitovanievpomesheniyach/.

[5] Indoor Positioning Systems based on BLE Beacons. URL: http://ja-si.com/ble-beacons/indoorpositioning-system/.

[6] A.V. Mezhenin, V.V. Izvozchikova, A.V. Kovalevsky, Technologies and methods of Indoor navigation systems, in: Proceedings of the. International scientific and practical conference, Prospects for the development of modern mathematical and natural sciences'2, Voronezh ,2015, pp. 100-103.

[7] Falkov E.V., A.Yu. Romanov, Application of Beacon beacons and Bluetooth Low Energy technology for building navigation systems in buildings, New information technologies in automated systems 18 (2015) 62-65.

[8] R.Yu. Safronov, Development of a mobile application for ios using "ibeacon" technology for solving problems of navigation inside buildings based on wireless networks, Engineering bulletin of the Don 4 (2014) 69.

[9] New iOS 7: iBeacon technology Apple's secret weapon. URL: macilove.com/news/new-ios-7technology-ibeacon-apple-ssecret-weapon/.

[10] M.S. Smolkov, Yu.A. Sukhobok, Analysis of modern technologies for building internal navigation systems, Scientific, technical and economic cooperation of the Asia-Pacific countries in the XXI century 2 (2019) 88-92.

[11] V.P. Kirnos, A.L. Priorov, Algorithms for object recognition on multidimensional television images for scene mapping in the SLAM problem, in: Proceedings of the 12th. international scientific and technical conference Advanced technologies in the means of information transmission, PTSPI-2017, Vladimir, 2017,pp. 126-128.

[12] Creation of an application for internal navigation based on ARCore in Unity. URL: https://blog.raccoons.be/arcore-powered-indoor-navigation-unity.

[13] Overcoming the problem of undefined tracking errors in the AR-navigation application. URL: https://github.com/Oscheibe/ARIndoorNav. 